\title{
Long-time behavior of a Cahn-Hilliard-Navier-Stokes vesicle-fluid interaction model
}

\author{
Blanca Climent Ezquerra \\ Francisco Guillén González
}

Cádiz, junio de 2015 


\section{The Model: Interaction of a vesicle membrane with a fluid field in 3D}

Evolution of vesicles immersed in an incompressible Newtonian fluid. Volume and area are conserved.

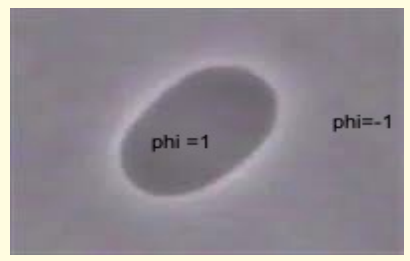

System:

- Navier-Stokes eqs.+stress term membrane.

- Incompressibility eq.

- Phase-field eq. of Cahn-Hilliard type (diffuse interface) 
$F(\phi)=\frac{1}{4}\left(\phi^{2}-1\right)^{2}$ Ginzburg-Landau potential,

$M, \alpha>0$ constants, $\quad \varepsilon$ interface width,

$A(\phi)=\int_{\Omega}\left(\frac{\varepsilon}{2}|\nabla \phi|^{2}+\frac{1}{\varepsilon} F^{\prime}(\phi)\right) d x$, area surface.

Bending energy

$$
E_{b}(\phi)=\frac{1}{2 \varepsilon} \int_{\Omega}\left(-\varepsilon \Delta \phi+\frac{1}{\varepsilon} F^{\prime}(\phi)\right)^{2} d x+\frac{1}{2} M(A(\phi)-\alpha)^{2}
$$

\section{Cahn-Hilliard equation}

$$
\partial_{t} \phi+\boldsymbol{u} \cdot \nabla \phi=-\Delta \frac{\delta E_{b}(\phi)}{\delta \phi}
$$


$F(\phi)=\frac{1}{4}\left(\phi^{2}-1\right)^{2}$ Ginzburg-Landau potential,

$M, \alpha>0$ constants, $\quad \varepsilon$ interface width,

$A(\phi)=\int_{\Omega}\left(\frac{\varepsilon}{2}|\nabla \phi|^{2}+\frac{1}{\varepsilon} F^{\prime}(\phi)\right) d x$, area surface.

Bending energy

$$
E_{b}(\phi)=\frac{1}{2 \varepsilon} \int_{\Omega}\left(-\varepsilon \Delta \phi+\frac{1}{\varepsilon} F^{\prime}(\phi)\right)^{2} d x+\frac{1}{2} M(A(\phi)-\alpha)^{2}
$$

\section{Cahn-Hilliard equation}

$$
\partial_{t} \phi+\boldsymbol{u} \cdot \nabla \phi=-\Delta \frac{\delta E_{b}(\phi)}{\delta \phi}
$$




\section{Other phase-field equation}

$B(\phi)=\int_{\Omega} \phi d x$, volume.

\section{Bending energy}

$$
\begin{gathered}
E_{b}(\phi)=\frac{1}{2 \varepsilon} \int_{\Omega}\left(-\varepsilon \Delta \phi+\frac{1}{\varepsilon} F^{\prime}(\phi)\right)^{2} d x+\frac{1}{2} M_{1}(A(\phi)-\alpha)^{2} \\
+\frac{1}{2} M_{2}(B(\phi)-\beta)^{2}
\end{gathered}
$$

\section{Allen-Cahn equation}

$$
\partial_{t} \phi+\boldsymbol{u} \cdot \nabla \phi=-\frac{\delta E_{b}(\phi)}{\delta \phi}
$$

Q. Du,M. Li,C. Liu'07 
$F(\phi)=\frac{1}{4}\left(\phi^{2}-1\right)^{2}$ Ginzburg-Landau potential,

$M, \alpha>0$ constants, $\quad \varepsilon$ interface width,

$A(\phi)=\int_{\Omega}\left(\frac{\varepsilon}{2}|\nabla \phi|^{2}+\frac{1}{\varepsilon} F^{\prime}(\phi)\right) d x$, area surface.

Bending energy

$$
E_{b}(\phi)=\frac{1}{2 \varepsilon} \int_{\Omega}\left(-\varepsilon \Delta \phi+\frac{1}{\varepsilon} F^{\prime}(\phi)\right)^{2} d x+\frac{1}{2} M(A(\phi)-\alpha)^{2}
$$

\section{Cahn-Hilliard equation}

$$
\partial_{t} \phi+\boldsymbol{u} \cdot \nabla \phi=-\Delta \frac{\delta E_{b}(\phi)}{\delta \phi}
$$


Chemical potential:

$$
w:=\frac{\delta E_{b}(\phi)}{\delta \phi}=\varepsilon \Delta^{2} \phi+G(\phi)
$$

where

$$
\begin{aligned}
G(\phi):= & -\frac{1}{\varepsilon} \Delta F^{\prime}(\phi)+\frac{1}{\varepsilon^{2}}\left(-\varepsilon \Delta \phi+\frac{1}{\varepsilon} F^{\prime}(\phi)\right) F^{\prime}(\phi) \\
& +M(A(\phi)-\alpha)\left(-\varepsilon \Delta \phi+\frac{1}{\varepsilon} F^{\prime}(\phi)\right) .
\end{aligned}
$$

Cahn-Hilliard equations

$$
\begin{aligned}
& \partial_{t} \phi+\boldsymbol{u} \cdot \nabla \phi-\Delta w=0, \\
& \varepsilon \Delta^{2} \phi+G(\phi)-w=0 .
\end{aligned}
$$




\section{The equations of the model}

Changing the variables

$$
\psi(x, t):=\phi(x, t)-m_{0} \text { and } z:=w-\langle w\rangle \text { with } m_{0}=\langle\phi(x, 0)\rangle
$$

\section{Navier-Stokes-Cahn-Hilliard system}

$$
\begin{aligned}
& \left(\partial_{t} \boldsymbol{u}+(\boldsymbol{u} \cdot \nabla) \boldsymbol{u}-\Delta \boldsymbol{u}-z \nabla \psi+\nabla \widetilde{\boldsymbol{q}}=0,\right. \\
& \nabla \cdot \boldsymbol{u}=0 \\
& \partial_{t} \psi+\boldsymbol{u} \cdot \nabla \psi-\Delta z=0, \\
& \varepsilon \Delta^{2} \psi+\bar{G}(\psi)-z=0 \text {, } \\
& \left.\boldsymbol{u}\right|_{\partial \Omega}=0,\left.\quad \partial_{n} \psi\right|_{\partial \Omega}=0,\left.\quad \partial_{n} \Delta \psi\right|_{\partial \Omega}=0,\left.\quad \partial_{n} z\right|_{\partial \Omega}=0, \\
& \boldsymbol{u}(0)=\boldsymbol{u}_{0}, \quad \psi(0)=\psi_{0}:=\phi(x, 0)-m_{0} \quad \text { in } \Omega .
\end{aligned}
$$

where

$$
\bar{G}(\psi):=G\left(\psi+m_{0}\right)-\left\langle G\left(\psi+m_{0}\right)\right\rangle,
$$




$$
\begin{gathered}
\boldsymbol{H}=\left\{\boldsymbol{u} \in \boldsymbol{L}^{2} ; \nabla \cdot \boldsymbol{u}=0, \boldsymbol{u} \cdot \mathbf{n}=0 \text { on } \partial \Omega\right\} \\
\boldsymbol{V}=\left\{\boldsymbol{u} \in \boldsymbol{H}^{\mathbf{1}} ; \nabla \cdot \boldsymbol{u}=0, \boldsymbol{u}=0 \text { on } \partial \Omega\right\}
\end{gathered}
$$

$$
\begin{aligned}
& L_{*}^{2}=\left\{v \in L^{2}(\Omega) ; \int_{\Omega} v=0\right\}, \\
& H_{*}^{k}=\left\{v \in H^{k}(\Omega) ; \int_{\Omega} v=0\right\} \quad k \geq 1, \\
& H_{1}^{2}=\left\{v \in H_{*}^{2}(\Omega) ;\left.\partial_{n} v\right|_{\partial \Omega}=0\right\} \\
& H_{2}^{k}=\left\{v \in H_{*}^{k} ;\left.\partial_{n} v\right|_{\partial \Omega}=0,\left.\partial_{n} \Delta v\right|_{\partial \Omega}=0\right\} \quad k=3,4,5,6 .
\end{aligned}
$$




$$
\begin{gathered}
E_{k}(\boldsymbol{u})=\frac{1}{2} \int_{\Omega}|\boldsymbol{u}|^{2}, \quad \bar{E}_{b}(\psi)=E_{b}\left(\psi+m_{0}\right) \\
\bar{E}(\boldsymbol{u}, \psi)=E_{k}(\boldsymbol{u})+\bar{E}_{b}(\psi)
\end{gathered}
$$

\section{Energy Inequality}

$$
\frac{d}{d t} \bar{E}(\boldsymbol{u}(t), \psi(t))+|\nabla \boldsymbol{u}(t)|_{2}^{2}+|\nabla z(t)|_{2}^{2} \leq 0 .
$$




\section{Global weak estimates}

$$
\begin{gathered}
\boldsymbol{u} \in L^{2}(0,+\infty ; \boldsymbol{V}) \cap L^{\infty}(0,+\infty ; \boldsymbol{H}), \quad z \in L^{2}\left(0,+\infty ; H_{*}^{1}\right) \\
\psi \in L^{\infty}\left(0,+\infty ; H_{1}^{2}\right) .
\end{gathered}
$$

\section{Additional estimate}

$$
\psi \in L_{l o c}^{2}\left(0,+\infty ; H_{2}^{5}\right)
$$




\section{Strong solution}

Strong estimates for velocity for large times

$$
\begin{gathered}
\boldsymbol{u} \in L^{\infty}\left(\left[T_{\text {reg }}^{*},+\infty\right) ; \boldsymbol{H}^{\boldsymbol{1}}\right) \cap L^{2}\left(\left[T_{\text {reg }}^{*},+\infty\right) ; \boldsymbol{H}^{2}\right), \\
\partial_{t} \boldsymbol{u} \in L^{2}\left(\left[T_{\text {reg }}^{*},+\infty\right) ; \boldsymbol{L}^{2}\right) .
\end{gathered}
$$

Global in time strong estimate for $\psi$

$$
\psi \in L^{\infty}\left(0,+\infty ; H_{2}^{3}\right) .
$$

Strong estimates for $\psi$ and $z$

$$
\begin{gathered}
\psi \in L_{\text {loc }}^{2}\left(0,+\infty ; H_{2}^{6}\right), \quad \partial_{t} \psi \in L_{\text {loc }}^{2}\left(0,+\infty ; L_{*}^{2}\right) \\
z \in L_{\text {loc }}^{2}\left(0,+\infty ; H_{1}^{2}\right) .
\end{gathered}
$$




\section{Convergence at infinite time of strong solution}

$$
\bar{E}(\boldsymbol{u}(t), \psi(t)) \searrow E_{\infty} \text { in } \mathbf{R} \quad \text { as } t \uparrow+\infty .
$$

\section{$\omega$-limit set}

Fixed a global weak solution, $(\boldsymbol{u}, \psi)$, associated to the initial data, $\left(\boldsymbol{u}_{0}, \psi_{0}\right) \in \boldsymbol{V} \times \boldsymbol{H}_{2}^{3}$,

$$
\begin{aligned}
\omega(\boldsymbol{u}, \psi)=\left\{\left(\boldsymbol{u}_{\infty}, \psi_{\infty}\right) \in \boldsymbol{V} \times \boldsymbol{H}_{2}^{3}: \exists\left\{t_{n}\right\} \uparrow+\infty\right. \text { s.t. } \\
\left.\left(\boldsymbol{u}\left(t_{n}\right), \psi\left(t_{n}\right)\right) \rightarrow\left(\boldsymbol{u}_{\infty}, \psi_{\infty}\right) \text { weakly in } \boldsymbol{H}^{1} \times \boldsymbol{H}_{2}^{3}\right\} .
\end{aligned}
$$

\section{Set of equilibrium points}

$$
\mathcal{S}=\left\{(0, \psi): \psi \in H_{2}^{4}(\Omega): \varepsilon \Delta^{2} \psi+\bar{G}(\psi)=0 \text { a.e in } \Omega\right\} .
$$


Assume that $\left(\boldsymbol{u}_{0}, \psi_{0}\right) \in \boldsymbol{V} \times H_{2}^{3}$, fixed $(\boldsymbol{u}(t), \psi(t), z(t))$ a weak solution in $(0,+\infty)$,

\section{Theorem}

$\omega(\boldsymbol{u}, \psi)$ is nonempty and $\omega(\boldsymbol{u}, \psi) \subset \boldsymbol{S}$.

For any $(0, \bar{\psi}) \in \omega(\boldsymbol{u}, \psi) \subset S, \bar{E}(\bar{\psi})=E_{\infty}$

\section{Theorem}

There exists $\bar{\psi} \in H_{2}^{4}$, such that $\psi(t) \rightarrow \bar{\psi}$ in $H_{2}^{3}$ weakly as $t \uparrow+\infty$, i.e.

$$
\omega(\boldsymbol{u}, \psi)=\{(0, \bar{\psi})\} .
$$


Tools of proof: BCE, F. Guillén-González'14.

- BCE, F. Guillén-González, M.A. Rodríguez-Bellido'10,

- Lemma of strong continuous dependence with respect to initial data.

- Lojasiewicz-Simon Lemma.

H. Wu, X. Xu'13: Navier-Stokes-Allen-Cahn 


\section{Lemma}

Let $\Phi, B \in L^{1}(0,+\infty)$ be two positive functions such that $\Phi \in H^{1}(0, T) \forall T>0$, which satisfies

$$
\Phi^{\prime}(t) \leq C\left(\Phi(t)^{3}+B(t)\right) .
$$

Then, there exists a sufficiently large $T^{*} \geq 0$ such that $\Phi \in L^{\infty}\left(T^{*},+\infty\right)$ and

$$
\exists \lim _{t \rightarrow+\infty} \Phi(t)=0
$$


Continuous dependence initial data

If $\left(\boldsymbol{u}^{\varepsilon}, \psi^{\varepsilon}, \boldsymbol{z}^{\varepsilon}\right)$, for some $\varepsilon>0$, and $\left(\boldsymbol{u}^{0}, \psi^{0}, z^{0}\right)$ are two regular solutions in $\left(0, T^{*}\right)$ of the problem; associated to the different initial conditions, $\left(\boldsymbol{u}_{0}^{\varepsilon}, \psi_{0}^{\varepsilon}\right) \in \boldsymbol{H}^{1} \times \boldsymbol{H}_{2}^{3}$ and $\left(\boldsymbol{u}_{0}, \psi_{0}\right) \in \boldsymbol{H}^{1} \times \boldsymbol{H}_{2}^{3}$, respectively, then $\boldsymbol{u}^{\varepsilon}-\boldsymbol{u}^{0}, \psi^{\varepsilon}-\psi^{0}$ and $z^{\varepsilon}-z^{0}$ depend continuously of the initial values in the following sense: If $\boldsymbol{u}_{0}^{\varepsilon} \rightarrow \boldsymbol{u}_{0}$ weakly in $\boldsymbol{H}^{1}$ (and strongly in $\boldsymbol{L}^{2}$ ) and $\psi_{0}^{\varepsilon} \rightarrow \psi_{0}$ weakly in $H_{2}^{3}$ (and strongly in $H_{1}^{2}$ ), then,

$$
\begin{array}{cc}
\boldsymbol{u}^{\varepsilon}-\boldsymbol{u}^{0} \rightarrow 0 & \text { in } L^{\infty}\left(0, T^{*} ; \boldsymbol{L}^{2}\right) \cap L^{2}\left(0, T^{*} ; \boldsymbol{H}^{1}\right), \\
\psi^{\varepsilon}-\psi^{0} \rightarrow 0 & \text { in } L^{\infty}\left(0, T^{*} ; H^{2}\right) \cap L^{2}\left(0, T^{*} ; H^{5}\right) .
\end{array}
$$




\section{Convergence at infinite time of strong solution}

\section{Lojasiewicz-Simon inequality}

Let $\mathcal{S}$ be the following set of equilibrium points related to the bending energy

$$
\mathcal{S}=\left\{\psi \in H_{2}^{4}(\Omega): \varepsilon \Delta^{2} \psi+\bar{G}(\psi)=0 \text { a.e in } \Omega\right\} .
$$

If $\bar{\psi} \in \mathcal{S}$, there are three positive constants $C, \alpha$, and

$\theta \in(0,1 / 2)$ which depend on $\bar{\psi}$, such that for all $\psi \in H_{2}^{4}$ and $\|\psi-\bar{\psi}\|_{2} \leq \beta$, then

$$
\left|\bar{E}_{b}(\psi)-\bar{E}_{b}(\bar{\psi})\right|^{1-\theta} \leq C|z|_{2}
$$

where $z=z(\psi):=\varepsilon \Delta^{2} \psi+\bar{G}(\psi)$. 


\section{$\omega$-limit set}

Fixed a global weak solution, $(\boldsymbol{u}, \psi)$, associated to the initial data, $\left(\boldsymbol{u}_{0}, \psi_{0}\right) \in \boldsymbol{H} \times H_{1}^{2}$,

$$
\begin{aligned}
\omega(\boldsymbol{u}, \psi)=\left\{\left(\boldsymbol{u}_{\infty}, \psi_{\infty}\right) \in \boldsymbol{H} \times H_{1}^{2}: \exists\left\{t_{n}\right\} \uparrow+\infty\right. \text { s.t. } \\
\left.\left(\boldsymbol{u}\left(t_{n}\right), \psi\left(t_{n}\right)\right) \rightarrow\left(\boldsymbol{u}_{\infty}, \psi_{\infty}\right) \text { weakly in } \boldsymbol{H} \times H_{1}^{2}\right\} .
\end{aligned}
$$




\section{Convergence at infinite time of weak solution}

Assume that $\left(\boldsymbol{u}_{0}, \psi_{0}\right) \in \boldsymbol{H} \times H_{1}^{2}$, fixed $(\boldsymbol{u}(t), \psi(t), z(t))$ a weak solution in $(0,+\infty)$,

\section{Theorem}

$\omega(\boldsymbol{u}, \psi)$ is nonempty and $\omega(\boldsymbol{u}, \psi) \subset \boldsymbol{S}$.

For any $(0, \bar{\psi}) \in \omega(\boldsymbol{u}, \psi) \subset S$ then $\bar{E}(\bar{\psi})=E_{\infty}$

\section{Theorem}

There exists $\bar{\psi} \in H_{2}^{4}$, such that $\psi(t) \rightarrow \bar{\psi}$ in $H_{1}^{2}$ weakly as $t \uparrow+\infty$, i.e.

$$
\omega(\boldsymbol{u}, \psi)=\{(0, \bar{\psi})\} .
$$


Tools of proof:

- H. Petzeltova, E. Rocca, G. Schimperna'13.

- Lojasiewicz-Simon Lemma. 
Consider the initial and boundary value problem associated to the problem on the time interval $\left[t_{n}, t_{n}+1\right]$ with initial values $\boldsymbol{u}\left(t_{n}\right)$ and $\psi\left(t_{n}\right)$. Define

$$
\begin{gathered}
\boldsymbol{u}_{n}(t):=\boldsymbol{u}\left(t+t_{n}\right) \\
\psi_{n}(t):=\psi\left(t+t_{n}\right) \\
z_{n}(t):=z\left(\psi\left(t+t_{n}\right)\right)
\end{gathered}
$$

for a.e.

$$
t \in[0,1],
$$

$\left(\boldsymbol{u}_{n}, \psi_{n}\right)$ is a weak solution on the time interval $[0,1]$. 


\section{Convergence at infinite time of strong solution}

Lojasiewicz-Simon inequality

Let $\mathcal{S}$ be the following set of equilibrium points related to the bending energy $\bar{E}_{b}(\psi)$

$$
\mathcal{S}=\left\{\psi \in H_{2}^{4}(\Omega): \varepsilon \Delta^{2} \psi+\bar{G}(\psi)=0 \text { a.e in } \Omega\right\} .
$$

Let $\bar{\psi} \in \mathcal{S}$ and $K>0$ fixed. Then, for any two sufficiently small constants $\beta$ and $\delta$, there exists $C>0$ and $\theta \in(0,1 / 2)$ (depending on $\bar{\psi}, \beta$ and $\delta$ ), such that for all $\psi \in H_{2}^{4}$ with $\|\psi\|_{3} \leq K,\|\psi-\bar{\psi}\|_{1} \leq \beta$ and $\left|\bar{E}_{b}(\psi)-\bar{E}_{b}(\bar{\psi})\right| \leq \delta$, it holds

$$
\left|\bar{E}_{b}(\psi)-\bar{E}_{b}(\bar{\psi})\right|^{1-\theta} \leq C|z|_{2}
$$

where $z=z(\psi):=\varepsilon \Delta^{2} \psi+\bar{G}(\psi)$. 